\title{
Molecular hydrogen at high-z: physical conditions in protogalaxies
}

\author{
R. Srianand ${ }^{1}$, P. Petitjean ${ }^{2}$, C. Ledoux ${ }^{3}$, G. Ferland ${ }^{4}$, and G. Shaw ${ }^{4}$ \\ ${ }^{1}$ IUCAA, Post Bag 4, Ganesh Khind, Pune 411 007, India - email: anand@iucaa.ernet.in \\ ${ }^{2}$ Institut d'Astrophysique de Paris - CNRS, 98bis Boulevard Arago, F-75014 Paris, France \\ ${ }^{3}$ European Southern Observatory, Alonso de Córdova 3107, \\ Casilla 19001, Vitacura, Santiago, Chile \\ ${ }^{4}$ Department of Physics and Astronomy, University of Kentucky, \\ 177 Chemistry/Physics Building, Lexington, KY 40506, USA
}

\begin{abstract}
We study the physical conditions in damped Lyman- $\alpha$ systems (DLAs), using a sample of 33 systems toward 26 QSOs acquired for a recently completed survey of $\mathrm{H}_{2}$ by Ledoux et al. (2003). $\mathrm{H}_{2}$ is detected in $13-20 \%$ of the DLAs in our sample. Using the rotation level populations of $\mathrm{H}_{2}$ and fine-structure excitations of $\mathrm{C}$ I we show the mean kinetic temperature of $\mathrm{H}_{2}$ components is $153 \pm 78 \mathrm{~K}, n_{\mathrm{H}}=10-250 \mathrm{~cm}^{-3}$, and the ambient radiation field is similar to or slightly higher than the mean diffuse UV field of the Galaxy. Combining this with the success rate of detecting $\mathrm{H}_{2}$ in DLAs we conclude that at least $13-20 \%$ of DLAs at $z_{\text {abs }} \geqslant 1.9$ show the presence of CNM and substantial star-formation activity. C II* absorption is detected in all the components where $\mathrm{H}_{2}$ absorption is seen. The level populations of $\mathrm{C}$ II in these systems is consistent with the physical parameters derived from the excitation of $\mathrm{H}_{2}$ and $\mathrm{C}$ I. We detect $\mathrm{C} \mathrm{II}^{*}$ in about $50 \%$ of the DLAs and therefore in a considerable fraction of DLAs that do not show $\mathrm{H}_{2}$. The absence of $\mathrm{C}$ I absorption, the measured $\mathrm{N}\left(\mathrm{C} \mathrm{II}^{*}\right) / \mathrm{N}(\mathrm{C}$ II $)$ and $\mathrm{N}(\mathrm{Al}$ III $) / \mathrm{N}(\mathrm{Al}$ II $)$ ratios in these systems are consistent with the gas having lower density $\left(\mathrm{n}_{\mathrm{H}}=0.3-6 \mathrm{~cm}^{-3}\right)$ than that seen in the $\mathrm{H}_{2}$ components. $50 \%$ of the DLAs that do not show $\mathrm{C} \mathrm{II}^{*}$ are consistent with them originating from a low density warm neutral medium.
\end{abstract}

\section{Introduction}

Damped Ly- $\alpha$ (DLA) systems seen in QSO spectra are characterised by very large neutral hydrogen column densities: $N(\mathrm{H} \mathrm{I}) \geqslant 2 \times 10^{20} \mathrm{~cm}^{-2}$. Such an amount of neutral gas is usually measured through local spiral disks. The case for DLA systems to arise through proto-galactic disks is further supported by the fact that the cosmological density of the absorbing gas at $z_{\text {abs }} \sim 3$ is of the same order of magnitude as the cosmological density of stars at present epochs (Wolfe 1995). $\mathrm{H}_{2}$ is ubiquitous in the neutral phase of the interstellar medium (ISM) of galaxies. Formation of $\mathrm{H}_{2}$ is expected on the surface of dust grains, if the gas is cool, dense, and mostly neutral, and from the formation of $\mathrm{H}^{-}$ ions if the gas is warm and dust-free. As the former process is most likely dominant in the neutral gas, it is possible to obtain an indirect indication of the dust content in DLAs without depending on extinction and/or heavy element depletion effects. Moreover, by determining the populations of different $\mathrm{H}_{2}$ rotational levels, it is possible to constrain the kinetic temperature and density through rotational excitation temperatures. Effective photo-dissociation of $\mathrm{H}_{2}$ takes place in the energy range $11.1-13.6 \mathrm{eV}$ through Lymanand Werner-band absorption lines and the intensity of the local UV radiation field can therefore be derived from the observed molecular fraction. A direct determination of the local radiation field and physical conditions of the gas will have important implications in bridging the link between DLA systems and star-formation activity at high redshifts. 
We have searched for $\mathrm{H}_{2}$ in DLA and sub-DLA systems at high redshift $\left(z_{\mathrm{abs}}>1.8\right)$, using UVES at the VLT down to a detection limit of typically $N\left(\mathrm{H}_{2}\right) \sim 2 \times 10^{14} \mathrm{~cm}^{-2}$ (see Ledoux et al. 2003). Out of the 33 systems in our sample, 8 have firm and 2 have tentative detections of associated $\mathrm{H}_{2}$ absorption lines. The systems where $\mathrm{H}_{2}$ is detected are usually among those with the highest metallicities and depletion factors. This directly demonstrates that a large amount of dust is present in the components where $\mathrm{H}_{2}$ is detected. The mean $\mathrm{H}_{2}$ molecular fraction $f=2 N\left(\mathrm{H}_{2}\right) /\left[2 N\left(\mathrm{H}_{2}\right)+N(\mathrm{HI})\right]$ in DLA systems is typically $\log f<-1$ and similar to what is observed in the Magellanic Clouds. There is no correlation between $f$ and $\mathrm{N}(\mathrm{H} \mathrm{I})$; in particular, two systems where $\mathrm{H}_{2}$ is detected have $\log N(\mathrm{HI})<20.3$. Approximately 50 percent of the systems have $\log f<$ -6. In this work, we estimate the range of physical conditions in the $\mathrm{H}_{2}$ components in DLAs using column densities of $\mathrm{H}_{2}$ in different rotational states, fine-structure excitation of $\mathrm{C}$ I and C II, N(S II), and standard techniques that are used in ISM studies. The details of the data used here can be found in Srianand \& Petitjean (1998), Petitjean et al. (2000, 2002), Srianand et al. (2000, 2005), and Ledoux et al. (2002, 2003).

\section{Rotational excitation of $\mathbf{H}_{2}$}

It is a standard procedure in ISM studies to use the $T_{01}$ obtained from

$$
\mathrm{OPR}_{\mathrm{LTE}} \sim \frac{\mathrm{N}(\mathrm{J}=1)}{\mathrm{N}(\mathrm{J}=0)}=9 \times \exp \left(-170.5 / \mathrm{T}_{01}\right)
$$

to infer the kinetic temperature of the gas assuming LTE. Panel (a) in Fig. 1 shows $\mathrm{T}_{01}$ measured in DLAs as a function of $\mathrm{N}\left(\mathrm{H}_{2}\right)$. The vertical dotted lines show the mean and $1 \sigma$ range of $T_{01}$ measured by Savage et al. (1977). $T_{01}=77 \pm 17 \mathrm{~K}$ measured by Savage et al. (1977) for the Galactic ISM is consistent with the mean temperature of the ISM measured using the H I $21 \mathrm{~cm}$ spin temperature, suggesting $T_{01}$ is a good tracer of the kinetic temperature in the optically thin $\mathrm{H}_{2}$. The data points from the Magellanic Clouds (Tumlinson et al. 2002) (with a mean $T_{01}=82 \pm 21 \mathrm{~K}$ ) are consistent with that seen in the Galactic ISM. However, most of the measurements from DLAs with optically thick $\mathrm{H}_{2}$ (i.e $\left.\log \mathrm{N}\left(\mathrm{H}_{2}\right) \geqslant 16.5\right)$ are well separated from that of the ISM and Magellanic Clouds (Fig. 1) and the spread seen in the optically thin case is consistent with that seen in the local ISM.

For the high optical depth clouds (i.e. $\log N\left(\mathrm{H}_{2}\right) \mathrm{cm}^{-2} \geqslant 16.5$ ) in DLAs the mean $T_{01}$ is $153 \pm 78 \mathrm{~K}$. In this high $\mathrm{N}\left(\mathrm{H}_{2}\right)$ range $T_{01}$ is expected to trace the kinetic temperature. Under the LTE assumption this will mean that the kinetic temperatures of the $\mathrm{H}_{2}$ components in DLAs are in the range 100 to $200 \mathrm{~K}$.

The rotational level populations are affected by particle collisions, UV pumping, and formation pumping. While collisional excitation plays a significant role in populating the low-J levels, those with $\mathrm{J} \geqslant 3$ are usually populated by formation processes and UV pumping. We can see from panel (b) in Fig. 1 that in DLAs where $\mathrm{H}_{2}$ is optically thick, the $N(\mathrm{~J}=2) / N(\mathrm{~J}=0)$ ratio is larger than that seen in similar gas of the Galactic ISM, LMC, and SMC. It is also clear that the excitation temperature is in the range 100 to $600 \mathrm{~K}$. We notice that the excitation temperatures $T_{02}$ and $T_{03}$ are consistent with one another (see panel (c) in Fig. 1) but higher than $T_{01}$ (see Srianand et al. 2005 for details). This clearly means $\mathrm{J} \geqslant 2$ levels are influenced by UV pumping and/or formation pumping.

Following the analytic prescription of Jura (1975) we can write

$$
p_{4,0} \beta(0) n\left(H_{2}, J=0\right)+0.24 R n(H) n=A(4 \rightarrow 2) n\left(H_{2}, J=4\right) .
$$

Here, $\beta(0)$ and $p_{4,0}$ are respectively the photo-absorption rate in the Lyman and Werner bands, and the pumping efficiency from $\mathrm{J}=0$ to $\mathrm{J}=4 ; \mathrm{A}(4 \rightarrow 2)$ is the spontaneous 

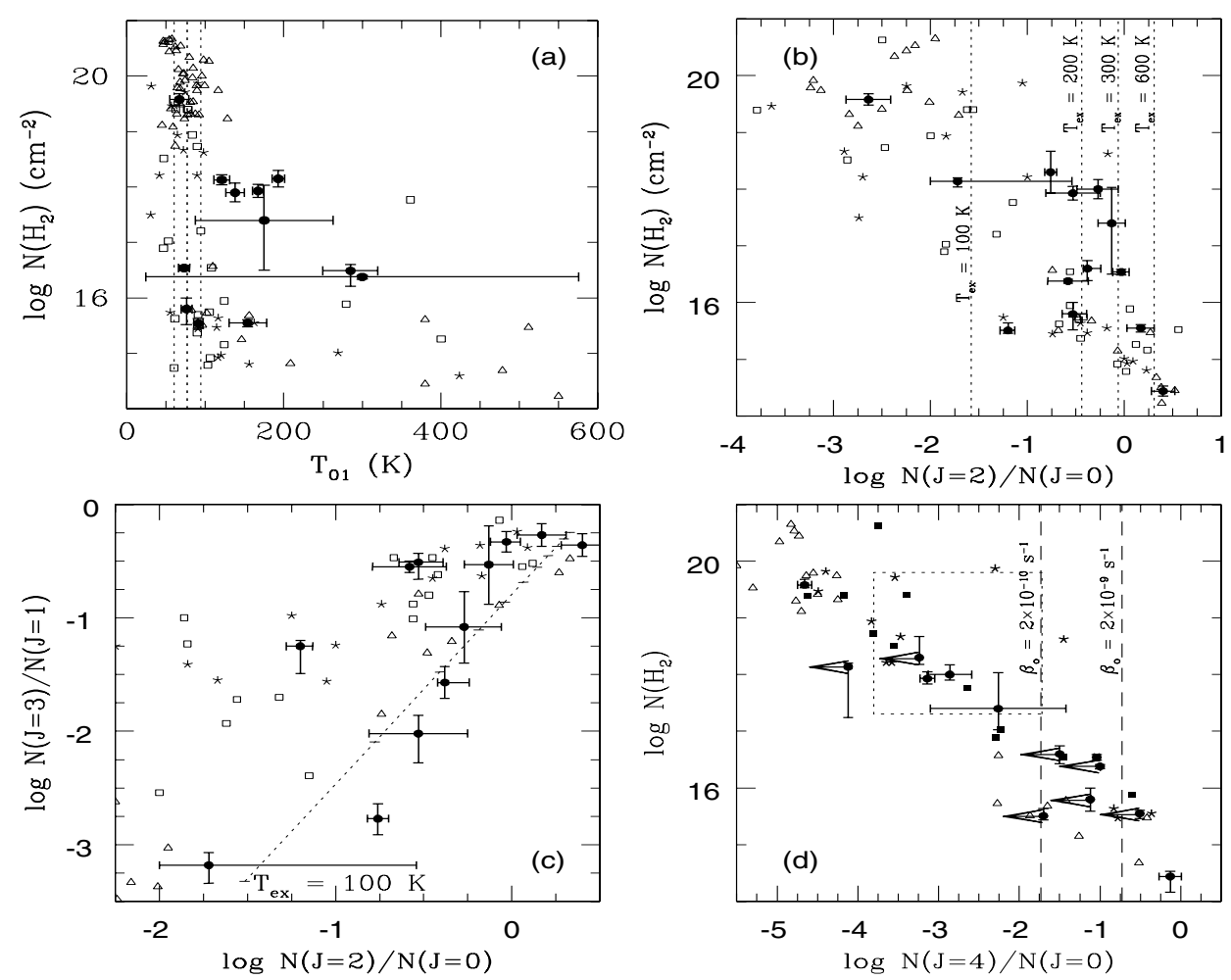

Figure 1. Filled circles with error-bars are our measurements in DLA components, other data points are from Savage et al. (1977), Spitzer, Cochran \& Hirshfelf (1974) for the Galactic ISM (triangles), and Tumlinson et al. (2002) for the LMC (squares) and SMC (asterisks). The vertical short-dashed lines in panel (a) show the mean and $1 \sigma$ range of $T_{01}$ measured by Savage et al. (1977) in the Galactic ISM. Vertical dashed lines in panels (b) give the expected ratio for different excitation temperatures. In panel (c) the dotted line gives the expected relation under LTE with temperatures ranging from 100 to $600 \mathrm{~K}$ (horizontal tick-marks show the values for different temperatures with $50 \mathrm{~K}$ steps). In panel (d) the vertical dashed lines give the predicted ratio for different values of photo-absorption rate $\beta_{0}$.

transition probability between $\mathrm{J}=4$ and $\mathrm{J}=2$; and $R$ is the formation rate of $\mathrm{H}_{2}$. Neglecting the second term in the left hand side of Eq. 2.2 leads to a conservative upper limit on the UV radiation field.

For $\log N\left(\mathrm{H}_{2}\right) \leqslant 16.5$ the $N(\mathrm{~J}=4) / N(\mathrm{~J}=0)$ ratio in DLAs is of the order of or slightly higher than that seen in the ISM of our Galaxy. Quantitatively the upper limits in most of the systems are consistent with $2 \times 10^{-10} \leqslant \beta(0) \leqslant 2 \times 10^{-9} \mathrm{~s}^{-1}$. This probably means the optically thin $\mathrm{H}_{2}$ components without detectable $\mathrm{H}_{2}$ absorption from the $\mathrm{J}=4$ state arise in gas embedded in a UV field with intensity similar to (or slightly higher than) that of the mean ISM field. Detailed analysis of two optically thin components $\left(z_{\mathrm{abs}}=1.96822\right.$ toward Q 0013-004 and 3.02489 toward Q 0347-383) in our sample suggests an ambient field intensity consistent with a few times the mean ISM field intensity (Petitjean et al. 2002; Levshakov et al. 2002). 

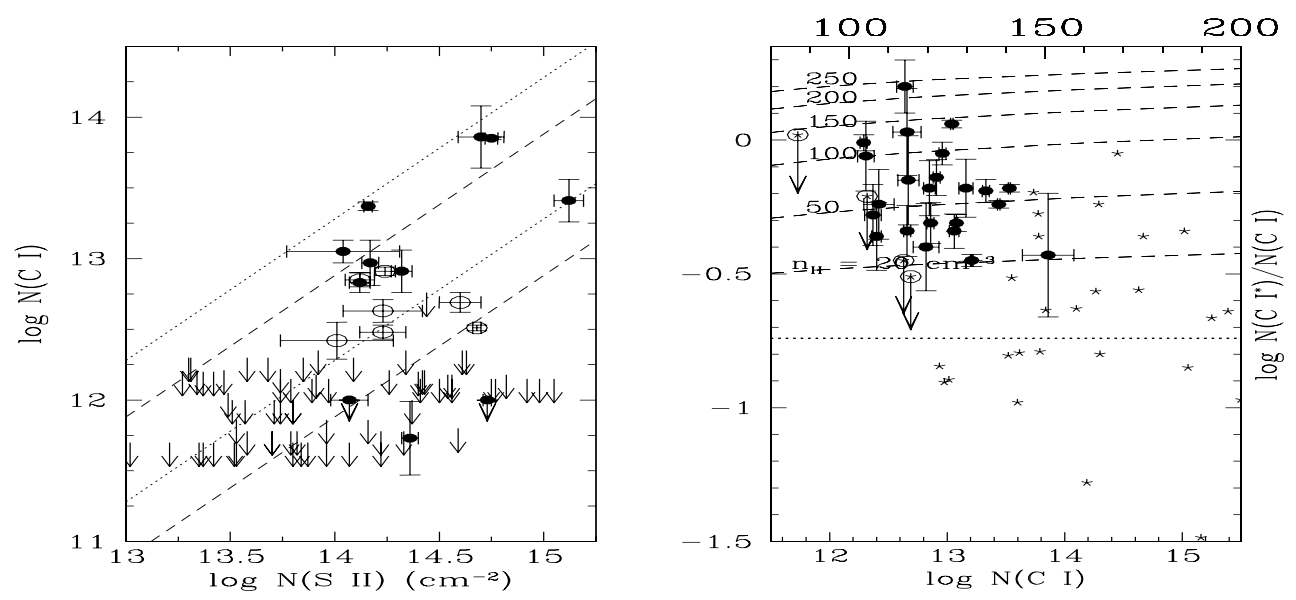

Figure 2. Left panel: N(C I) measured in individual components of DLAs (filled and open circles are for the components with and without $\mathrm{H}_{2}$ detection respectively) as a function N(S II). The arrows are the upper limits from systems where there is no $\mathrm{H}_{2}$ or $\mathrm{C}$ I absorption. The two dotted lines give the expected correlation for $\log N(\mathrm{C}$ I $) / N(\mathrm{C}$ II $)=-3$ (lower line) and -2 (upper line) respectively, when Solar relative abundances are used. The short-dashed lines give the same correlations when $\mathrm{C}$ is depleted with respect to $\mathrm{S}$ by $0.4 \mathrm{dex}$. The $\mathrm{C}$ I detections are consistent with what one expects in the case of the CNM. Right panel: The ratio $N\left(\mathrm{C} \mathrm{I}^{*}\right) / N(\mathrm{C} \mathrm{I})$ is plotted as a function of $N(\mathrm{C} \mathrm{I})$. The points with error bars are our measurements in individual DLA components. The stars are data measurements in the ISM of the Milky Way drawn from Jenkins \& Tripp (2001) and Jenkins, Jura \& Loewenstein (1983). The horizontal dotted line gives the expected value of the ratio if it is assumed that $\mathrm{C}_{\mathrm{I}}$ is excited by the CMBR only with $T_{\mathrm{CMBR}}$ $=8.1 \mathrm{~K}$, as expected at $z=2$. The short dashed lines give the expected ratio for different $n_{\mathrm{H}}$ as a function of temperature (top portion of the $\mathrm{x}$-axis).

\section{C I absorption}

$\mathrm{C}$ I is usually a good tracer of the physical conditions in $\mathrm{H}_{2}$ gas as the ionisation state of carbon is sensitive to the same photons that destroy $\mathrm{H}_{2}$ (see however Srianand \& Petitjean 1998). Usually, DLAs in which no $\mathrm{H}_{2}$ is detected through the whole profile do not show any detectable $\mathrm{C}$ I absorption (with a typical upper limit of $10^{12} \mathrm{~cm}^{-2}$ ). The only exception is the high-metallicity sub-DLA at $z_{\text {abs }}=2.139$ toward Tol 1037-270 (see Srianand \& Petitjean 2001). On the contrary, in DLAs where $\mathrm{H}_{2}$ is detected, some components show detectable $\mathrm{C}$ I absorption without detectable $\mathrm{H}_{2}$ absorption $\left(N\left(\mathrm{H}_{2}\right) \leqslant 10^{14} \mathrm{~cm}^{-2}\right)$. This is the case in Q 0013-004 (Petitjean et al. 2002) and Q 0551-366 (Ledoux et al. 2002). In the case of the $z_{\mathrm{abs}}=2.495$ system toward $\mathrm{Q} 0405-443 \mathrm{H}_{2}$ is detected in two components without strong $\mathrm{C}$ I absorption (Ledoux et al. 2003).

Among the systems that show $\mathrm{H}_{2}$ absorption there is no clear trend between $N\left(\mathrm{H}_{2}\right)$ and $N(\mathrm{C}$ I $)$. We notice detectability of $\mathrm{C}$ I absorption is highly probable in systems with high dust depletion and metallicity (Srianand et al. 2005). We show, using N(S II) as a proxy for $\mathrm{N}(\mathrm{C}$ II $)$, that the DLA components with $\mathrm{C}$ I detections have an ionisation state consistent with them originating from the cold neutral medium (CNM) (see Fig. 2). The distribution of $N(\mathrm{~S} \mathrm{II})$ is somewhat similar for components with both $\mathrm{H}_{2}$ and $\mathrm{C}$ I absorptions (filled circles), and for components with $\mathrm{C}$ I but no $\mathrm{H}_{2}$ absorptions (open circles). However, $\mathrm{N}(\mathrm{C} \mathrm{I})$ in components without $\mathrm{H}_{2}$ are typically lower. Most of the upper limits on $\mathrm{C}$ I are consistent with $N(\mathrm{C}$ I $) / N(\mathrm{C}$ II $) \leqslant-3$. This can mean most of 


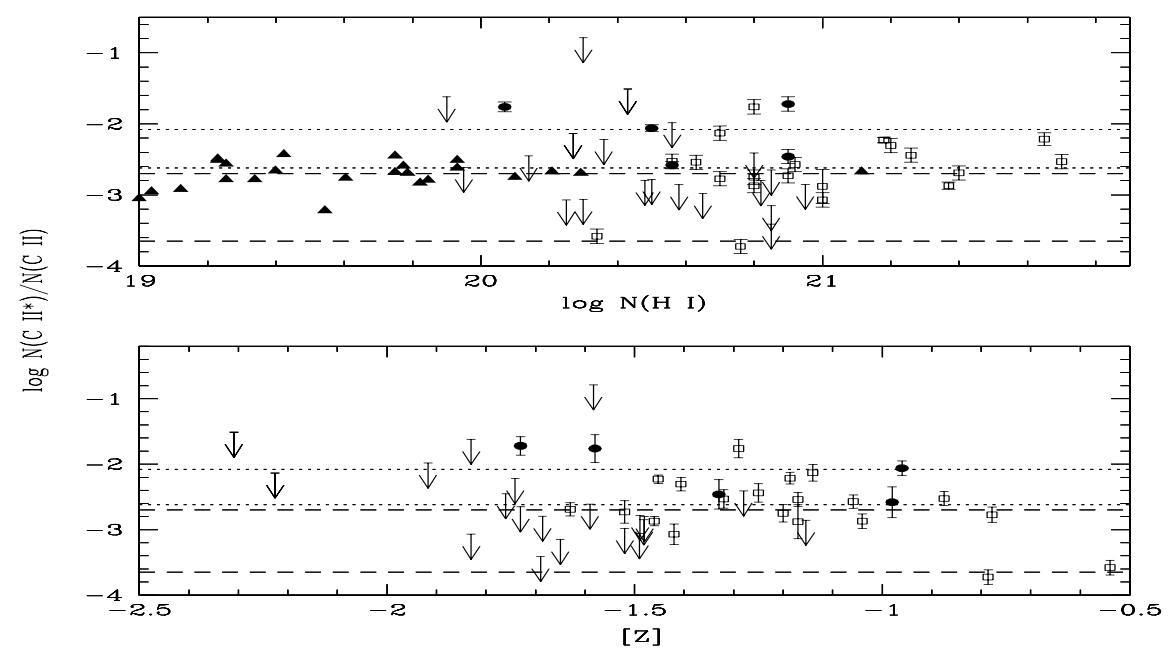

Figure 3. The average (over the whole profile) $N\left(\mathrm{C} \mathrm{II}^{*}\right) / N(\mathrm{C}$ II) ratio in DLAs (combining our sample to measures by Wolfe et al. 2003a, 2003b) is plotted against $\log N$ (H I) (upper panel) and the metallicity $\mathrm{Z}$ (lower panel). The dotted lines show the expected range of values observed in the CNM gas with metallicity and dust-content ranging from that observed in the ISM to that of typical DLAs. The dashed lines gives the corresponding range for the WNM gas. Filled circles and open squares are respectively for systems with and without $\mathrm{H}_{2}$ detections. In the top panel the filled triangles are the observations of Lehner et al. (2004) along high latitude Galactic sight-lines with low $\mathrm{H}_{2}$ content. Most of the points with $\log N(\mathrm{H} \mathrm{I}) \leqslant 20.0$ are from intermediate or high velocity clouds in the Galactic halo.

the DLA systems originate from warm neutral medium (WNM) or warm ionised medium (WIM) where the above ratio can be as low as $10^{-4}$. The CMBR field expected from the Big Bang is not sufficient to explain the observed $\mathrm{N}\left(\mathrm{C} \mathrm{I}^{*}\right) / \mathrm{N}(\mathrm{C} \mathrm{I})$ and an extra contribution is required from collisional processes and/or the UV flux (right panel in Fig. 2). For the temperature range seen in $\mathrm{H}_{2}$ components from $T_{01}$ and a UV radiation field like that seen in the Galactic ISM, the observed $\mathrm{N}\left(\mathrm{C} \mathrm{I}^{*}\right) / \mathrm{N}(\mathrm{C} \mathrm{I})$ is consistent with $20 \leqslant n_{H}\left(\mathrm{~cm}^{-3}\right) \leqslant 250$.

\section{C II* absorption}

All the systems with $\mathrm{H}_{2}$ detections showed $\mathrm{C}$ II* absorption. $\mathrm{C}$ II absorption is also detected in a considerable fraction of DLAs that do not show $\mathrm{H}_{2}$ or $\mathrm{C}$ I absorption. However $50 \%$ of the DLAs do not show detectable $\mathrm{C}$ II* absorption. In the case of systems with $\mathrm{H}_{2}$ detections $N\left(\mathrm{C} \mathrm{II}^{*}\right) / N(\mathrm{C}$ II $)$ is consistent with that expected in the CNM (see Fig. 3). From the upper panel in Fig. 3 it can be seen that $\mathrm{C}$ II* is detected in all the systems with $\log N(\mathrm{H} \mathrm{I}) \geqslant 21.0$. Most the systems with $\log N(\mathrm{H} \mathrm{I}) \geqslant 21.0$ have $N\left(\mathrm{C}\right.$ II $\left.^{*}\right) / N(\mathrm{C}$ II $)$ consistent with what is expected in CNM. On the contrary, the measured values of $N\left(\mathrm{C}\right.$ II $\left.^{*}\right) / N(\mathrm{C}$ II $)$ in systems with lower $N(\mathrm{H} \mathrm{I})$ spread over more than an order of magnitude, covering the expected ranges for WNM and CNM. From the bottom panel, it can be seen that C II* is frequently detected in gas with high metallicity, as already noticed by Wolfe et al. (2003a). In the whole sample the number of systems with $\mathrm{C}$ II* without $\mathrm{H}_{2}$ detections that are consistent with a $\mathrm{CNM}$ and a WNM are approximately equal. However using $\mathrm{N}(\mathrm{Al}$ III $) / \mathrm{N}(\mathrm{Al}$ II $)$ and the absence of $\mathrm{H}_{2}$ for the depletion inferred in the systems, we show the values of $n_{H}$ in these systems are probably 
lower than that seen in the components with $\mathrm{H}_{2}$ detection. Most of the upper limits on $\mathrm{N}\left(\mathrm{C}\right.$ II $\left.^{*}\right) / \mathrm{N}(\mathrm{C}$ II $)$, measured in the metallicity range $-2.0 \leqslant Z_{\odot} \leqslant-1.5$, are lower than what would be expected from CNM gas and are consistent with WNM (or low density) gas. Interestingly these upper limits are lower than that seen in high latitude Galactic sight lines that are believed to be predominantly WNM gas. This means that the electron density (and total particle density) in these DLAs is probably quite small.

\section{Conclusions}

$\mathrm{H}_{2}$ absorption is detected in $13-20 \%$ of the DLAs that also show fine-structure lines of C I and C II. We show that in these systems $75 \leqslant \mathrm{~T}(\mathrm{~K}) \leqslant 230,20 \leqslant n_{H} \leqslant 250 \mathrm{~cm}^{-3}$, and the radiation field is of the order of or slightly higher than the mean local ISM UV field. The physical state of the gas is similar to that expected from a CNM. If the inferred radiation field persists in $20 \%$ of the DLA population the star-formation rate in DLAs can appreciably contribute to the global star-formation rate density at high- $z$. $50 \%$ of the DLAs in our sample do not show either atomic fine-structure lines or $\mathrm{H}_{2}$. In addition $\mathrm{C}$ I absorption lines are not detected. These systems are consistent with the absorption originating from low density gas. In our sample, roughly $30 \%$ of the DLAs show $\mathrm{C} \mathrm{II}^{*}$ absorption without showing $\mathrm{H}_{2}$ or $\mathrm{C} \mathrm{I} . \mathrm{n}_{H}$ is probably lower than what is typically seen in the $\mathrm{H}_{2}$ components.

\section{Acknowledgements}

Results presented in this work are based on observations carried out at ESO under programmes ID No. 65.P-0038, 65.O-0063, 66.A-0624, 67.A-0078, 68.A-0600, 68.A-0106, and 70.A-0017 with the UVES on VLT. RS and PPJ gratefully acknowledge support from the IFCPAR under contract No. 3004-3. GJF and RS acknowledge support from the DST/INT/US(NSF-RP0-115)/2002.

\section{References}

Jenkins, E. B., Jura, M., Loewenstein, M., 1983, ApJ, 270, 1

Jenkins, E. B., Tripp, T. M., 2001, ApJ, 137, 297

Jura, M., 1975, ApJ, 197, 575

Ledoux, C., Petitjean, P., Srianand, R., 2003, MNRAS, 346, 209

Ledoux, C., Srianand, R., Petitjean, P., 2002, A\&A 392, 781

Lehner, N., Wakker, B. P., Savage, B. D., 2004, astro-ph/0407363

Levshakov, S. A., Dessauges-Zavadsky, M., D’Odorico, S., Molaro, P., 2002, ApJ, 565, 696

Petitjean, P., Srianand, R., Ledoux, C., 2000, A\&A, 364, L26

Petitjean, P., Srianand, R., Ledoux, C., 2002, MNRAS, 332, 383

Savage, B. D., Drake, J. F., Budich, W., Bohlin, R. C., 1977, ApJ, 216, 291

Spitzer, L. Jr., Cochran, W. D., Hirshfeld, A., 1974, ApJS, 28, 373

Srianand, R., Petitjean, P., 1998, A\&A, 335, 33

Srianand, R., Petitjean, P., 2001, A\&A, 373, 816

Srianand, R., Petitjean, P., Ledoux, C., 2000, Nature, 408, 931

Srianand, R., Petitjean, P., Ledoux, C., Ferland, G., Shaw, G., 2005, MNRAS, preprint.

Tumlinson, J., Shull, J. M., Rachford, B. L., et al., 2002, ApJ, 566, 857

Wolfe A. M., 1995, in QSO Absorption Lines, Proc. ESO Workshop, ed. G. Meylan (Berlin: Springer), p. 13

Wolfe, A. M., Gawiser, E., Prochaska, J. X., 2003b, ApJ, 593, 235

Wolfe, A. M., Prochaska, J. X., Gawiser, E., 2003a, ApJ, 593, 215 